Noname manuscript No.

(will be inserted by the editor)

\title{
B-Nekrasov matrices and error bounds for linear complementarity problems
}

\author{
M. García-Esnaola · Juan Manuel Peña
}

Received: date / Accepted: date

\begin{abstract}
The class of $B$-Nekrasov matrices is a subclass of $P$-matrices that contains Nekrasov $Z$-matrices with positive diagonal entries as well as $B$-matrices. Error bounds for the linear complementarity problem when the involved matrix is a $B$-Nekrasov matrix are presented. Numerical examples show the sharpness and applicability of the bounds.
\end{abstract}

Keywords Error bounds - Linear complementarity problems · Nekrasov matrices · $B$-matrices $\cdot B$-Nekrasov matrices

Mathematics Subject Classification (2000) 90C33 · 90C31 - 65G50 · 15A48

\section{Introduction}

The linear complementarity problem $(L C P)$ has a crucial importance in many applications, as shown in [1]. It looks for vectors $x \in R^{n}$ such that

$$
A x+q \geq 0, \quad x \geq 0, \quad x^{T}(A x+q)=0
$$

where $A$ is an $n \times n$ real matrix and $q \in R^{n}$. This problem will be denoted by $\operatorname{LCP}(A, q)$ and its solutions by $x^{*}$. It presents nice properties when the matrix $A$ belongs to some special classes of matrices. If $A$ is a $P$-matrix, then the $L C P$ problem has a unique solution and formulae for the error bound can be provided (see [3], [4], [12] and Section 3). If $A$ satisfies the stronger property of being an $H$-matrix with positive diagonal entries, then the error bound becomes simpler (see formula (2.4) of [3]). In the particular case that $A$ belongs to certain

\footnotetext{
M. García-Esnaola

Departamento de Matemática Aplicada

Universidad de Zaragoza

Tel.: (+34) 976761985

E-mail: gesnaola@unizar.es

Juan Manuel Peña

Departamento de Matemática Aplicada

Universidad de Zaragoza

Tel.: (+34) 976761129

E-mail: jmpena@unizar.es
} 
subclasses of $H$-matrices, more formulae for error bounds can be provided (see [9], and [11] for Nekrasov matrices). When the matrix $A$ of (1) is not an $H$-matrix we cannot use formula (2.4) of [3]. However, for some subclasses of $P$-matrices that are not $H$-matrices, error bounds for the $L C P$ problem have also been obtained. For instance, for $B$-matrices ([8]), for $D B$-matrices ([6]), for $S B$-matrices ([7]), for $B^{S}$-matrices ([10]) and for $M B$-matrices ([2]).

In this paper we present an error bound for a class of $P$-matrices containing as subclasses both $B$-matrices and Nekrasov $Z$-matrices. We call these matrices as $B$-Nekrasov matrices.

In Section 2, we define the classes of matrices mentioned in this paper and we analyze the $B$-Nekrasov matrices. We prove that they are $P$-matrices and we present a characterization of the $B$-Nekrasov matrices. In Section 3, we obtain error bounds for linear complementarity problems corresponding to $B$-Nekrasov matrices. We also include numerical experiments that show the sharpness and applicability of the bounds.

\section{B-Nekrasov matrices}

We start by introducing some classes of matrices. A square real matrix with nonpositive off-diagonal entries is called a Z-matrix. The unisolvence of the $\operatorname{LCP}(A, q)$ problem given in (1) holds if and only if $A$ is a $P$-matrix (see [4]), concept that will be recalled now. A submatrix of a square matrix involving the same rows and columns is called a principal submatrix. A real square matrix is a $P$-matrix if all its principal minors are positive. A complex matrix $A=\left(a_{i j}\right)_{1 \leq i, j \leq n}$ is strictly diagonally dominant (by rows) if $\left|a_{i i}\right|>\sum_{j \neq i}\left|a_{i j}\right|$, for all $i=1, \ldots, n$. A $Z$-matrix is a nonsingular $M$-matrix if it has nonnegative inverse. A square complex matrix $A$ is an $H$-matrix if there exists a diagonal matrix $X$ such that $A X$ is strictly diagonally dominant. It is well known that nonsingular $M$-matrices and $H$-matrices with positive diagonal entries are $P$-matrices. In order to define Nekrasov matrices, let us introduce some notations. Given a complex matrix $A=\left(a_{i j}\right)_{1 \leq i, j \leq n}$ with $a_{i i} \neq 0$ for all $i=1, \ldots, n$, we define

$$
h_{1}(A):=\sum_{j \neq 1}\left|a_{1 j}\right|, h_{i}(A):=\sum_{j=1}^{i-1}\left|a_{i j}\right| \frac{h_{j}(A)}{\left|a_{j j}\right|}+\sum_{j=i+1}^{n}\left|a_{i j}\right|, \quad i=2, \ldots, n .
$$

Then we say that $A$ is a Nekrasov matrix if $\left|a_{i i}\right|>h_{i}(A)$ for all $i=1, \ldots, n$ (cf. [5], [11], [15]). It is known that a Nekrasov matrix is an $H$-matrix (see page 5021 of [5] and [15]). From now on whenever a reference to $H$-matrices (resp., Nekrasov matrices) is made it will mean real matrices as the LCP requires. We finish this list of known definitions with the concept of $B$-matrix (see [13]). We say that a square real matrix $A=\left(a_{i j}\right)_{1 \leq i, j \leq n}$ with positive row sums is a $B$-matrix if all its off-diagonal entries are (strictly) bounded above by the corresponding row means, that is, for each $i=1, \ldots, n$,

$$
\sum_{k=1}^{n} a_{i k}>0, \quad \frac{1}{n} \sum_{k=1}^{n} a_{i k}>a_{i j}, \forall j \neq i
$$

In contrast to Nekrasov matrices, $B$-matrices are not necessarily $H$-matrices.

Let us now define a class of matrices that will contain Nekrasov $Z$-matrices with positive diagonal entries as well as $B$-matrices. First we recall a decomposition of square matrices that will be useful for our purposes.

Given a real matrix $A=\left(a_{i j}\right)_{1 \leq i, j \leq n}$, we can write it as

$$
A=B^{+}+C
$$


where

$$
B^{+}=\left(\begin{array}{rrr}
a_{11}-r_{1}^{+} & \ldots & a_{1 n}-r_{1}^{+} \\
\vdots & \vdots \\
\vdots & \vdots \\
a_{n 1}-r_{n}^{+} & \ldots & a_{n n}-r_{n}^{+}
\end{array}\right) \text {and } C=\left(\begin{array}{rrr}
r_{1}^{+} & \ldots & r_{1}^{+} \\
\vdots & \vdots \\
\vdots & \vdots \\
r_{n}^{+} & \ldots & r_{n}^{+}
\end{array}\right)
$$

with

$$
r_{i}^{+}:=\max \left\{0, a_{i j} \mid j \neq i\right\} .
$$

Observe that $B^{+}$is a $Z$-matrix and $C$ is a nonnegative matrix of rank 1 .

Definition 1 We say that $A$ is a $B$-Nekrasov matrix if it can be written in form (3) with $B^{+}$a Nekrasov $Z$-matrix whose diagonal entries are all positive. For this to happen, the maximum of the positive off-diagonal elements of each row must be strictly less than the corresponding positive diagonal element of the original matrix.

Remark 1 Let us observe that a Nekrasov $Z$-matrix with positive diagonal entries is trivially a $B$-Nekrasov matrix (with $C=0$ in (3)) and that a $B$-matrix is also a $B$-Nekrasov matrix. This last property is a consequence from the fact that a strictly diagonally dominant matrix is a Nekrasov matrix and from Proposition 2.1 of [8], which characterizes $B$-matrices through (3), with the condition that $B^{+}$is a strictly diagonally dominant (by rows) matrix with positive diagonal entries.

The following family of matrices belongs to the class of $B$-Nekrasov matrices although they are not Nekrasov matrices nor $B$-matrices:

$$
A_{k}=\left(\begin{array}{rrrr}
4 & 3 & 3 & 3 \\
-k & 3 & 1 & 1 \\
-k & 1 & 5 & 0 \\
-k & 1 & 0 & 4
\end{array}\right), \quad k \geq 2
$$

Observe that matrices $A_{k}$ are not Nekrasov because the first row is not strictly diagonally dominant. The decomposition (3) for these matrices becomes

$$
A_{k}=B_{k}^{+}+C_{k}=\left(\begin{array}{rrrr}
1 & 0 & 0 & 0 \\
-k-1 & 2 & 0 & 0 \\
-k-1 & 0 & 4 & -1 \\
-k-1 & 0 & -1 & 3
\end{array}\right)+\left(\begin{array}{llll}
3 & 3 & 3 & 3 \\
1 & 1 & 1 & 1 \\
1 & 1 & 1 & 1 \\
1 & 1 & 1 & 1
\end{array}\right)
$$

and satisfies Definition 1. Finally, matrices $A_{k}$, for $k \geq 2$, are not $B$-matrices because matrices $B_{k}^{+}$are not strictly diagonally dominant matrices. Let us see now that $B$-Nekrasov matrices are $P$-matrices.

Proposition 1 If A is a B-Nekrasov matrix, then A is a P-matrix.

Proof By Definition 1, $A=B^{+}+C$, where $B^{+}$is a Nekrasov $Z$-matrix and $C$ is a nonnegative matrix of rank 1. It is well-known that a Nekrasov matrix is a nonsingular $H$-matrix (cf. [5]) and so $B^{+}$is a nonsingular $M$-matrix. Then the result follows from Corollary 2.4 of [14], which guarantees that $A$ is a $P$-matrix because it is the sum of a nonsingular $M$-matrix and a nonnegative matrix of rank 1

Let us provide a characterization of $B$-Nekrasov matrices. 
Theorem 1 Let $A=\left(a_{i j}\right)_{1 \leq i, j \leq n}$ be a real matrix. Then $A$ is $B$-Nekrasov if and only if the following conditions hold:

(i) $\sum_{j=1}^{n} a_{1 j}>0$ and $\frac{1}{n}\left(\sum_{j=1}^{n} a_{1 j}\right)>a_{1 k}$, for all $k \neq 1$.

(ii) $r_{i}^{+}<\min \left\{\frac{\sum_{j=1}^{i-1} a_{i j} \alpha_{j}+\sum_{j=i}^{n} a_{i j}}{\sum_{j=1}^{i-1} \alpha_{j}+(n-i+1)}, a_{i i}\right\}, i=2, \ldots, n$, where $r_{i}^{+}$is given in (5), $\alpha_{1}:=\frac{\sum_{j=2}^{n}\left|a_{1 j}-r_{1}^{+}\right|}{\left|a_{11}-r_{1}^{+}\right|}$ and $\alpha_{i}:=\frac{\sum_{j=1}^{i-1}\left|a_{i j}-r_{i}^{+}\right| \alpha_{j}+\sum_{j=i+1}^{n}\left|a_{i j}-r_{i}^{+}\right|}{\left|a_{i i}-r_{i}^{+}\right|}$for $i=2, \ldots, n$.

Proof Let us assume that (i) and (ii) hold, and let us see that the matrix $B^{+}$of (3) is a Nekrasov matrix. Given a matrix $A$, let $h_{i}(A)$ given by (2). Taking into account the definition of $r_{1}^{+}$and (i), we have: $h_{1}\left(B^{+}\right)=\sum_{j=2}^{n}\left|a_{1 j}-r_{1}^{+}\right|=\sum_{j=2}^{n}\left(r_{1}^{+}-a_{1 j}\right)=(n-1) r_{1}^{+}-\sum_{j=2}^{n} a_{1 j}=$ $\left(n r_{1}^{+}-\sum_{j=1}^{n} a_{1 j}\right)+\left(a_{11}-r_{1}^{+}\right)<a_{11}-r_{1}^{+}=\left|a_{11}-r_{1}^{+}\right|$and the first row of $B^{+}$satisfies the definition of a Nekrasov matrix. We continue with any row $i \geq 2$. Then

$$
\begin{gathered}
(0 \leq) h_{i}\left(B^{+}\right)=\sum_{j=1}^{i-1}\left|a_{i j}-r_{i}^{+}\right| \alpha_{j}+\sum_{j=i+1}^{n}\left|a_{i j}-r_{i}^{+}\right|=\sum_{j=1}^{i-1}\left(r_{i}^{+}-a_{i j}\right) \alpha_{j}+\sum_{j=i+1}^{n}\left(r_{i}^{+}-a_{i j}\right)= \\
=r_{i}^{+}\left(\sum_{j=1}^{i-1} \alpha_{j}+n-i+1\right)-\sum_{j=1}^{i-1} a_{i j} \alpha_{j}-r_{i}^{+}-\sum_{j=i}^{n} a_{i j}+a_{i i}= \\
=\left[r_{i}^{+}\left(\sum_{j=1}^{i-1} \alpha_{j}+n-i+1\right)-\sum_{j=1}^{i-1} a_{i j} \alpha_{j}-\sum_{j=i}^{n} a_{i j}\right]+\left(a_{i i}-r_{i}^{+}\right)<a_{i i}-r_{i}^{+}=\left|a_{i i}-r_{i}^{+}\right|
\end{gathered}
$$

and $B^{+}$is a Nekrasov matrix.

Conversely, let us now assume that $A$ is a $B$-Nekrasov matrix and let us prove that (i) and (ii) hold. Since $B^{+}$is a Nekrasov matrix with positive diagonal entries, we have

$$
a_{11}-r_{1}^{+}=\left|a_{11}-r_{1}^{+}\right|>\sum_{j=2}^{n}\left|a_{1 j}-r_{1}^{+}\right|=\sum_{j=2}^{n}\left(r_{1}^{+}-a_{1 j}\right)=(n-1) r_{1}^{+}-\sum_{j=2}^{n} a_{1 j}
$$

and so, $n r_{1}^{+}<\sum_{j=1}^{n} a_{1 j}$. In particular, $\sum_{j=1}^{n} a_{1 j}>0$ and, in addition, $r_{1}^{+}<\frac{1}{n} \sum_{j=1}^{n} a_{1 j}$ then condition (i) holds. Since $B^{+}$is a Nekrasov matrix with positive diagonal entries, $\left|a_{i i}-r_{i}^{+}\right|=$ $a_{i i}-r_{i}^{+}$and we also have

$$
\begin{array}{r}
a_{i i}-r_{i}^{+}=\left|a_{i i}-r_{i}^{+}\right|>h_{i}\left(B^{+}\right)=\sum_{j=1}^{i-1}\left|a_{i j}-r_{i}^{+}\right| \alpha_{j}+\sum_{j=i+1}^{n}\left|a_{i j}-r_{i}^{+}\right|= \\
=\sum_{j=1}^{i-1}\left(r_{i}^{+}-a_{i j}\right) \alpha_{j}+\sum_{j=i+1}^{n}\left(r_{i}^{+}-a_{i j}\right)=\sum_{j=1}^{i-1} r_{i}^{+} \alpha_{j}-\sum_{j=1}^{i-1} a_{i j} \alpha_{j}+(n-i) r_{i}^{+}-\sum_{j=i+1}^{n} a_{i j} .
\end{array}
$$

Then

$$
a_{i i}>r_{i}^{+}\left(\sum_{j=1}^{i-1} \alpha_{j}+(n-i+1)\right)-\sum_{j=1}^{i-1} a_{i j} \alpha_{j}-\sum_{j=i+1}^{n} a_{i j}
$$

and so

$$
r_{i}^{+}\left(\sum_{j=1}^{i-1} \alpha_{j}+(n-i+1)\right)<\sum_{j=1}^{i-1} a_{i j} \alpha_{j}+\sum_{j=i}^{n} a_{i j}
$$

Therefore,

$$
r_{i}^{+}<\frac{\sum_{j=1}^{i-1} a_{i j} \alpha_{j}+\sum_{j=i}^{n} a_{i j}}{\sum_{j=1}^{i-1} \alpha_{j}+(n-i+1)}
$$

and so (ii) holds. 


\section{Error bounds for linear complementarity problems}

In previous papers, we have obtained error bounds for linear complementarity problems when the matrix is a $B$-matrix (see [8]) and when the matrix is a Nekrasov matrix satisfying an additional property (see [11]). Since $B$-Nekrasov matrices contain $B$-matrices and Nekrasov $Z$-matrices, we consider in this section $B$-Nekrasov matrices such that $B^{+}$in (3) satisfies the mentioned additional property. We start with an auxiliary result for these matrices.

Lemma 1 Let $A=\left(a_{i j}\right)_{1 \leq i, j \leq n}, n \geq 2$, be a B-Nekrasov matrix such that for each $i=1, \ldots, n-$ 1 , there exists $m>i$ with $a_{i m}<\max \left\{0, a_{i j} \mid j \neq i\right\}=r_{i}^{+}$. Let $B^{+}$be the matrix of (3). Then the matrix $W=\operatorname{diag}\left(w_{1}, \ldots, w_{n}\right)$ with $w_{i}:=\frac{h_{i}\left(B^{+}\right)}{a_{i i}-r_{i}^{+}}$, for $i=1, \ldots, n-1$ and $w_{n}:=\frac{h_{n}\left(B^{+}\right)}{a_{n n}-r_{n}^{+}}+\varepsilon, \varepsilon \in$ $\left(0,1-\frac{h_{n}\left(B^{+}\right)}{a_{n n}-r_{n}^{+}}\right)$, has positive diagonal entries less than 1 and it satisfies that $B^{+} W$ is a strictly diagonally dominant Z-matrix.

Proof Observe that $B^{+}$is a Nekrasov matrix. Since $a_{i m}<r_{i}^{+}$, we have that $B^{+}$satisfies the hypotheses of Theorem 1 of [11] and, applying this theorem to $B^{+}$, the result follows.

The next lemma provides a typical bound for the inverse of certain matrices, and it will be used later.

Lemma 2 If $P:=\left(p_{1}, \ldots, p_{n}\right)^{T} e$, where $e=(1, \ldots, 1)$ and $p_{1}, \ldots, p_{n} \geq 0$, then

$$
\left\|(I+P)^{-1}\right\|_{\infty} \leq n-1,
$$

where I is the $n \times n$ identity matrix.

Proof Observe that

$$
(I+P)^{-1}=\left(\begin{array}{rrrr}
1-\frac{p_{1}}{1+\sum_{i=1}^{n} p_{i}} & -\frac{p_{1}}{1+\sum_{i=1}^{n} p_{i}} \ldots & -\frac{p_{1}}{1+\sum_{i=1}^{n} p_{i}} \\
-\frac{p_{2}}{1+\sum_{i=1}^{n} p_{i}} & 1-\frac{p_{2}}{1+\sum_{i=1}^{n} p_{i}} \ldots & -\frac{p_{2}}{1+\sum_{i=1}^{n} p_{i}} \\
\vdots & \vdots & \vdots \\
\vdots & \vdots & \vdots \\
-\frac{p_{n}}{1+\sum_{i=1}^{n} a_{i}} & -\frac{p_{n}}{1+\sum_{i=1}^{n} p_{i}} \ldots & 1-\frac{p_{n}}{1+\sum_{i=1}^{n} p_{i}}
\end{array}\right)
$$

Then we obtain

$$
\left\|(I+P)^{-1}\right\|_{\infty}=1+\frac{(n-2) \max _{i} p_{i}}{1+\sum_{i=1}^{n} p_{i}} \leq n-1
$$

and equality in the second relation above holds if and only if $n=2$.

The following result provides the announced error bounds for linear complementarity problems. By Proposition 1 a $B$-Nekrasov matrix is a $P$-matrix. Given an $n \times n P$-matrix $A$ and any $x \in \mathbf{R}^{n}$, by Theorem 2.3 of [3] we know that the solution $x^{*}$ of the linear complementarity problem (1) satisfies

$$
\left\|x-x^{*}\right\|_{\infty} \leq \max _{d \in[0,1]^{n}}\left\|(I-D+D A)^{-1}\right\|_{\infty}\|r(x)\|_{\infty},
$$


where $I$ is the $n \times n$ identity matrix, $D$ is the diagonal matrix $D=\operatorname{diag}\left(d_{i}\right)$ with $0 \leq d_{i} \leq 1$ for all $i=1, \ldots, n$, and $r(x):=\min (x, A x+q)$, where the min operator denotes the componentwise minimum of two vectors. The next result gives an upper bound for $\max _{d \in[0,1]^{n}} \|(I-$ $D+D A)^{-1} \|_{\infty}$.

Theorem 2 Let $A=\left(a_{i j}\right)_{1 \leq i, j \leq n}, n \geq 2$, be a B-Nekrasov matrix satisfying the hypotheses of Lemma 1 , let $B^{+}$be the matrix of (3) and let $W=\operatorname{diag}\left(w_{1}, \ldots, w_{n}\right)$ be the diagonal matrix of Lemma 1, such that $\bar{B}:=B^{+} W=\left(\bar{b}_{i j}\right)_{1 \leq i, j \leq n}$ is a strictly diagonally dominant Z-matrix. Let $\beta_{i}:=\bar{b}_{i i}-\sum_{j \neq i}\left|\bar{b}_{i j}\right|$ and $\delta_{i}:=\frac{\beta_{i}}{w_{i}}$ for $i=1, \ldots, n$, and $\delta:=\min _{i \in\{1, \ldots, n\}}\left\{\delta_{i}\right\}$. Then

$$
\max _{d \in[0,1]^{n}}\left\|(I-D+D A)^{-1}\right\|_{\infty} \leq \frac{(n-1) \max _{\mathrm{i}}\left\{w_{i}\right\}}{\min \{\delta, 1\} \min _{\mathrm{i}}\left\{w_{i}\right\}}
$$

Proof Since $A$ is a $B$-Nekrasov matrix, $A=B^{+}+C$ as in (3), with $B^{+}$being a Nekrasov $Z$-matrix with positive diagonal entries. Then $A W=\bar{B}+C W$, where $\bar{B}$ is strictly diagonally dominant by Lemma 1 and has positive diagonal entries. Given a diagonal matrix $D=\operatorname{diag}\left(d_{i}\right)$, with $0 \leq d_{i} \leq 1$, we have $A_{D}:=I-D+D A=\left(I-D+D B^{+}\right)+D C=B_{D}^{+}+C_{D}$, where $B_{D}^{+}:=I-D+D B^{+}$and $C_{D}:=D C$. Besides, we can write

$$
B_{D}^{+}=I-D+D\left(\bar{B} W^{-1}\right)=W\left(I-D+D\left(W^{-1} \bar{B}\right)\right) W^{-1} .
$$

Observe by Lemma 1 that $I-D+D\left(W^{-1} \bar{B}\right)$ is a strictly diagonally dominant $Z$-matrix and has positive diagonal entries. Therefore, $I-D+D\left(W^{-1} \bar{B}\right)$ is a nonsingular $M$-matrix and so, by Theorem 2.3 of Chapter 6 of [1], has nonnegative inverse. Since we can write $A_{D}=B_{D}^{+}\left(I+\left(B_{D}^{+}\right)^{-1} C_{D}\right), A_{D}^{-1}=\left(I+\left(B_{D}^{+}\right)^{-1} C_{D}\right)^{-1}\left(B_{D}^{+}\right)^{-1}$ and then

$$
\left\|A_{D}^{-1}\right\|_{\infty} \leq\left\|\left(I+\left(B_{D}^{+}\right)^{-1} C_{D}\right)^{-1}\right\|_{\infty}\left\|\left(B_{D}^{+}\right)^{-1}\right\|_{\infty} .
$$

Above, we have seen that $I-D+D\left(W^{-1} \bar{B}\right)$ has nonnegative inverse since $W$ is a positive diagonal matrix and then $\left(B_{D}^{+}\right)^{-1}=W\left(I-D+D\left(W^{-1} \bar{B}\right)\right)^{-1} W^{-1} \geq 0$. Observe that the matrix $C$ of (3) is nonnegative and with the form given in (4). Then $C_{D}$ is also nonnegative and with the form $C_{D}=\left(d_{1} r_{1}^{+}, \ldots, d_{n} r_{n}^{+}\right)^{T} e$, where $e=(1, \ldots, 1)$. Therefore $\left(B_{D}^{+}\right)^{-1} C_{D}$ can be writen as $\left(p_{1}, \ldots, p_{n}\right)^{T} e$, where $p_{i} \geq 0$ for all $i=1, \ldots, n$. By Lemma 2 we get

$$
\left\|\left(I+\left(B_{D}^{+}\right)^{-1} C_{D}\right)^{-1}\right\|_{\infty} \leq n-1 .
$$

As for the matrix $B_{D}^{+}$, we use (7) and, applying Theorem 1 of [16] to the strictly diagonally dominant matrix $I-D+D\left(W^{-1} \bar{B}\right)$, we obtain

$$
\left\|\left(B_{D}^{+}\right)^{-1}\right\|_{\infty} \leq \frac{\|W\|_{\infty}\left\|W^{-1}\right\|_{\infty}}{\min _{i}\left\{\bar{\alpha}_{i}^{D}\right\}}
$$

where $\bar{\alpha}_{i}^{D}:=\left(1-d_{i}\right)+\frac{d_{i}}{w_{i}}\left(\bar{b}_{i i}-\sum_{j \neq i}\left|\bar{b}_{i j}\right|\right)=\left(1-d_{i}\right)+\frac{d_{i}}{w_{i}} \beta_{i}(>0)$, for each $i=1, \ldots, n$. Observe that

$$
\|W\|_{\infty}=\max _{\mathrm{i}}\left\{w_{i}\right\} \quad \text { and } \quad\left\|W^{-1}\right\|_{\infty}=\frac{1}{\min _{i}\left\{w_{i}\right\}} .
$$

Let $k \in\{1, \ldots, n\}$ be the index with $\bar{\alpha}_{k}^{D}:=\min _{i}\left\{\bar{\alpha}_{i}^{D}\right\}$. Then

$$
\frac{1}{\min _{i}\left\{\bar{\alpha}_{i}^{D}\right\}}=\frac{1}{\left(1-d_{k}\right)+d_{k} \delta_{k}} \leq \frac{1}{\left(1-d_{k}\right)+d_{k} \delta} .
$$


If $\delta \geq 1$, then

$$
\frac{1}{\left(1-d_{k}\right)+d_{k} \delta} \leq 1
$$

If $\delta<1$, then

$$
\frac{1}{\left(1-d_{k}\right)+d_{k} \delta}=\frac{1}{1-(1-\delta) d_{k}} \leq \frac{1}{1-(1-\delta)}=\frac{1}{\delta} .
$$

By (12), (13) and (14) we have that

$$
\frac{1}{\min _{i}\left\{\bar{\alpha}_{i}^{D}\right\}} \leq \frac{1}{\min \{\delta, 1\}},
$$

and the result follows from (8), (9), (10), (11) and (15).

Let us illustrate the previous bound with a $B$-Nekrasov matrix that does not belong to classes of matrices for which error bounds are known.

Example 1 Let $A$ be the matrix

$$
A=\left(\begin{array}{rrrr}
60 & 20 & 20 & 30 \\
10 & 50 & -20 & 10 \\
-60 & 0 & 60 & -10 \\
30 & 30 & 20 & 40
\end{array}\right)
$$

We can check that $A$ is not an $H$-matrix, so that we cannot use the bounds valid for $H$ matrices. Since Nekrasov matrices and $S$-Nekrasov matrices are $H$-matrices, we cannot use the bounds of [11]. On the other hand, $A$ can be written $A=B^{+}+C$ as in (3), with

$$
B^{+}=\left(\begin{array}{rrrr}
30 & -10 & -10 & 0 \\
0 & 40 & -30 & 0 \\
-60 & 0 & 60 & -10 \\
0 & 0 & -10 & 10
\end{array}\right), \quad C=\left(\begin{array}{rrrr}
30 & 30 & 30 & 30 \\
10 & 10 & 10 & 10 \\
0 & 0 & 0 & 0 \\
30 & 30 & 30 & 30
\end{array}\right)
$$

Since $B^{+}$is not strictly diagonally dominant by rows, $A$ is not a $B$-matrix and so we cannot apply the bounds of [8]. By Lemma 2.5 of [10], $A$ is not a $B^{S}$-matrix and so we cannot apply the bounds of [10]. However, $B^{+}$is a Nekrasov matrix and so $A$ is $B$-Nekrasov. The diagonal matrix $W$ of Lemma 1 is given by $W=\operatorname{diag}\left(\frac{2}{3}, \frac{3}{4}, \frac{5}{6}, \frac{5}{6}+\varepsilon\right)$, with $\varepsilon \in\left(0, \frac{1}{6}\right)$. If we take $\varepsilon=\frac{1}{12}$, then $\delta=\frac{10}{11}$ and the bound (6) is 4.5375 .

We now consider $B$-Nekrasov matrices that are also $H$-matrices in order to compare our error bounds with formula (5) of [3]. Let us recall this last formula. Given a matrix $A=\left(a_{i j}\right)_{1 \leq i, j \leq n}$, we consider its comparison matrix $\tilde{A}=\left(\tilde{a}_{i j}\right)_{1 \leq i, j \leq n}$ with $\tilde{a}_{i i}:=\left|a_{i i}\right|$ for all $i=1, \ldots, n$, and $\tilde{a}_{i j}:=-\left|a_{i j}\right|$ when $j \neq i$. Then, by formula (5) of [3], the solution $x^{*}$ of the $L C P$ given by (1.1) for an $H$-matrix $A$ with positive diagonal entries satisfies

$$
\max _{d \in[0,1]^{n}}\left\|(I-D+D A)^{-1}\right\|_{\infty} \leq\left\|\tilde{A}^{-1} \max (\Lambda, I)\right\|_{\infty}
$$

with $\Lambda:=\operatorname{diag}\left(a_{i i}\right)$ and $\max (\Lambda, I):=\operatorname{diag}\left(\max \left\{a_{11}, 1\right\}, \ldots, \max \left\{a_{n n}, 1\right\}\right)$.

The following examples show two families of $2 \times 2$ and $3 \times 3 H$-matrices for which our bound (6) is close to 1 and 8 , respectively, in contrast to (16), which can be arbitrarily large. 
Example 2 Let $A_{k}$ be the $H$-matrices given by $A_{k}=\left(\begin{array}{rr}k-k+1 \\ -k+1 & k\end{array}\right), k \geq 2$. These matrices are also $B$-Nekrasov matrices with $A_{k}=B_{k}^{+}+C_{k}$ as in (3), $B_{k}^{+}=A_{k}$ and $C_{k}=0$. Then we have that $A_{k}=\tilde{A_{k}}$ and

$$
{\tilde{A_{k}}}^{-1}=\left(\begin{array}{cc}
\frac{k}{2 k-1} & \frac{k-1}{2 k-1} \\
\frac{k-1}{2 k-1} & \frac{k}{2 k-1}
\end{array}\right) \text {. }
$$

Then the matrix ${\tilde{A_{k}}}^{-1} \max (\Lambda, I)$ of $(16)$ is $\left(\begin{array}{cc}\frac{k^{2}}{2 k-1} & \frac{(k-1) k}{2 k-1} \\ \frac{(k-1) k}{2 k-1} & \frac{k^{2}}{2 k-1}\end{array}\right)$ and $\left\|\tilde{A}_{k}^{-1} \max (\Lambda, I)\right\|_{\infty}=k$. Therefore, the bound (16) can be arbitrarily large. Now we consider the bound (6). We have that $w_{1}=\frac{k-1}{k}, w_{2}=\left(\frac{k-1}{k}\right)^{2}+\varepsilon$, with $\varepsilon \in\left(0, \frac{2}{k}-\frac{1}{k^{2}}\right)$. The matrix $W$ of Theorem 1 is here

$$
W=\left(\begin{array}{lr}
\frac{k-1}{k} & 0 \\
0 & \left(\frac{k-1}{k}\right)^{2}+\varepsilon
\end{array}\right)
$$

and

$$
B_{k}^{+} W=\left(\begin{array}{rr}
k-1 & (-k+1)\left(\left(\frac{k-1}{k}\right)^{2}+\varepsilon\right) \\
\frac{(-k+1)(k-1)}{k} & k\left(\left(\frac{k-1}{k}\right)^{2}+\varepsilon\right)
\end{array}\right)
$$

If we take $\varepsilon=\frac{1}{k}$, we have that $\delta_{1}=\frac{k-1}{k}$ and $\delta_{2}=\frac{k^{2}}{k^{2}-k+1}$. Then $\delta=\min \left\{\delta_{1}, \delta_{2}\right\}=\frac{k-1}{k}$, $\max \left\{w_{1}, w_{2}\right\}=\frac{k^{2}-k+1}{k^{2}}, \min \left\{w_{1}, w_{2}\right\}=\frac{k-1}{k}$ and the corresponding bound of formula (6) is $1+\frac{k}{k^{2}-k+1}$, which converges to 1 when $k \rightarrow \infty$.

Example 3 Let $A_{k}$ be the $H$-matrices given by

$$
A_{k}=\left(\begin{array}{rrr}
k+1 & 0 & -k \\
0 & 2 k & -k \\
-k & 0 & k
\end{array}\right), \quad k \geq 4,
$$

with $A_{k}=B_{k}^{+}+C_{k}$ as in (3), $B_{k}^{+}=A_{k}, C_{k}=0$. Then $A_{k}=\tilde{A_{k}}$ and $\left\|{\tilde{A_{k}}}^{-1} \max (\Lambda, I)\right\|_{\infty}=2 k+2$. Therefore, the bound (16) can be arbitrarily large. Now we are going to obtain the bound (6). We have that $w_{1}=\frac{k}{k+1}, w_{2}=\frac{1}{2}, w_{3}=\frac{k}{k+1}+\varepsilon$, where $\varepsilon \in\left(0, \frac{1}{k+1}\right)$. The matrix $W$ of Theorem 1 is $W=\operatorname{diag}\left(\frac{k}{k+1}, \frac{1}{2}, \frac{k}{k+1}+\varepsilon\right)$ and

$$
B_{k}^{+} W=\left(\begin{array}{rrr}
k & 0 & -k\left(\frac{k}{k+1}+\varepsilon\right) \\
0 & k & -k\left(\frac{k}{k+1}+\varepsilon\right) \\
\frac{-k^{2}}{k+1} & 0 & k\left(\frac{k}{k+1}+\varepsilon\right)
\end{array}\right) .
$$

If we take $\varepsilon=\frac{1}{2 k}$, we have that $\beta_{1}=\beta_{2}=\frac{k-1}{2(k+1)}$ and $\beta_{3}=\frac{1}{2}$. Then $\delta_{1}=\frac{k-1}{2 k}, \delta_{2}=\frac{k-1}{k+1}$ and $\delta_{3}=\frac{k(k+1)}{2 k^{2}+k+1}$. Then $\delta=\min \left\{\delta_{1}, \delta_{2}, \delta_{3}\right\}=\frac{k-1}{2 k}, \max \left\{w_{1}, w_{2}, w_{3}\right\}=\frac{2 k^{2}+k+1}{2 k(k+1)}, \min \left\{w_{1}, w_{2}, w_{3}\right\}=$ $\frac{1}{2}$ and the corresponding bound of (6) is $\frac{8 k^{2}+4 k+4}{k^{2}-1}$, which converges to 8 when $k \rightarrow \infty$.

We now provide an alternative bound to (6) without using $\min _{i}\left\{w_{i}\right\}$ and $\max _{i}\left\{w_{i}\right\}$.

Proposition 2 Let $A=\left(a_{i j}\right)_{1 \leq i, j \leq n}, n \geq 2, B^{+}$and $W=\operatorname{diag}\left(w_{1}, \ldots, w_{n}\right)$ be the matrices of Lemma 1 and let $\delta$ be the real number defined in Theorem 2. Let $\gamma:=\min _{i \in\{1, \ldots, n-1\}} \frac{\sum_{j=i+1}^{n}\left|a_{i j}-r_{i}^{+}\right|}{a_{i i}-r_{i}^{+}}$. If either

$$
\sum_{j=1}^{n-1}\left(a_{n j}-r_{n}^{+}\right)=0
$$


holds or

$$
\sum_{j=1}^{n-1}\left|a_{n j}-r_{n}^{+}\right| \geq a_{n n}-r_{n}^{+}
$$

holds, then

$$
\max _{d \in[0,1]^{n}}\left\|(I-D+D A)^{-1}\right\|_{\infty}<\frac{n-1}{\min \{\delta, 1\} \gamma} .
$$

Otherwise, the following bound holds:

$$
\max _{d \in[0,1]^{n}}\left\|(I-D+D A)^{-1}\right\|_{\infty}<\frac{n-1}{\min \{\delta, 1\} \gamma} \frac{a_{n n}-r_{n}^{+}}{\sum_{j=1}^{n-1}\left|a_{n j}-r_{n}^{+}\right|} .
$$

Proof Observe that, by Lemma 1,

$$
1>w_{i} \geq \frac{\sum_{j=i+1}^{n}\left|a_{i j}-r_{i}^{+}\right|}{a_{i i}-r_{i}^{+}} \geq \gamma, \quad i=1, \ldots, n-1
$$

If (17) holds, then we can choose by Lemma 1 any $w_{n} \in(0,1)$. Since $\gamma<1$ by (21), we can take $w_{n}>\gamma$. So, by Lemma 1, (21) and Theorem 2, (19) holds. Now assume that (17) does not hold. From Lemma 1 we also derive $w_{n}>\sum_{j=1}^{n-1} \frac{\left|a_{n j}-r_{n}^{+}\right|}{a_{n n}-r_{n}^{+}} w_{j}$. Using (21) in the previous formula, we get $w_{n}>\gamma \sum_{j=1}^{n-1} \frac{\left|a_{n j}-r_{n}^{+}\right|}{a_{n n}-r_{n}^{+}}$and so

$$
\min _{i}\left\{w_{i}\right\} \geq \min \left\{\gamma, \gamma \sum_{j=1}^{n-1} \frac{\left|a_{n j}-r_{n}^{+}\right|}{a_{n n}-r_{n}^{+}}\right\}
$$

By Lemma 1, Theorem 2 and (22) we have

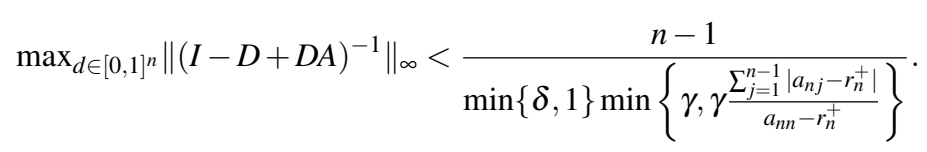

Observe that $\min \left\{\gamma, \gamma \frac{\sum_{j=1}^{n-1}\left|a_{n j}-r_{n}^{+}\right|}{a_{n n}-r_{n}^{+}}\right\}=\gamma\left(\right.$ respectively, $\gamma \frac{\sum_{j=1}^{n-1}\left|a_{n j}-r_{n}^{+}\right|}{a_{n n}-r_{n}^{+}}$) when (18) holds (resp., (18) does not hold). Now, (19) follows from (23) when (18) holds and (20) follows from (23) when (18) does not hold.

Remark 2 Observe that condition (17) of the previous theorem holds if and only if either the last row of the matrix $B^{+}$has zero entries up to the diagonal entry or $r_{n}^{+}=a_{n k}$ for all $k \in\{1,2, \ldots, n-1\}$. Besides, condition (18) holds if and only if the last row of the matrix $B^{+}$is not strictly diagonally dominant.

Acknowledgements The authors wish to thank the anonymous referees for their valuable suggestions to improve the paper. This work was partially supported by the Spanish Research Grant MTM2012-31544, Gobierno de Aragón and Fondo Social Europeo. 


\section{References}

1. A. Berman and R. J. Plemmons, Nonnegative matrices in the mathematical sciences, Classics in Applied Mathematics 9, SIAM, Philadelphia (1994).

2. T. Chen, W. Li, X. Wu, S. Vong, Error bounds for linear complementarity problems of $M B$-matrices. To appear in Numer. Algor. (DOI 10.1007/s11075-014-9950-9).

3. X. Chen and S. Xiang, Computation of error bounds for P-matrix linear complementarity problems, Math. Program., Ser. A, 106, 513-525 (2006).

4. R. W. Cottle, J.-S. Pang and R. E. Stone, The Linear Complementarity Problems, Academic Press, Boston MA (1992).

5. L. Cvetković, P-F. Dai, K. Doroslovaški, Y-T. Li, Infinity norm bounds for the inverse of Nekrasov matrices, Appl. Math. Comput., 219, 5020-5024 (2013).

6. P.-F. Dai, Error bounds for linear complementarity problems of DB-matrices, Linear Algebra Appl., 434, 830-840 (2011).

7. P.-F.Dai, Ch.-J. Lu, Y.-T. Li, New error bounds for the linear complementarity problem with an SB-matrix, Numer. Algor. 64, 741-757 (2013).

8. M. García-Esnaola and J. M. Peña, Error bounds for linear complementarity problems for B-matrices, Appl. Math. Lett. 22, 1071-1075 (2009).

9. M. García-Esnaola and J. M. Peña, A comparison of error bounds for linear complementarity problems of H-matrices, Linear Algebra Appl. 433, 956-964 (2010).

10. M. García-Esnaola and J. M. Peña, Error bounds for linear complementarity problems of $B^{S}$-matrices, Appl. Math. Lett., 25, 1379-1383 (2012).

11. M. García-Esnaola and J. M. Peña, Error bounds for linear complementarity problems of Nekrasov matrices, Numer. Algor., 67, 655-667 (2014).

12. R. Mathias and J.-S. Pang, Error bounds for the linear complementarity problem with a $P$-matrix, Linear Algebra Appl., 132, 123-136 (1990).

13. J. M. Peña, A class of $P$-matrices with applications to the localization of the eigenvalues of a real matrix, SIAM J. Matrix Anal. Appl., 22, 1027-1037 (2001).

14. J. M. Peña, On an alternative to Gerschgorin circles and ovals of Cassini, Numer. Math. 95, 337-345 (2003).

15. T. Szulc, Some remarks on a theorem of Gudkov, Linear Algebra Appl., 225, 221-235 (1995).

16. J. M. Varah, A Lower Bound for the Smallest Singular Value of a Matrix, Linear Algebra Appl., 11, 3-5 (1975). 